\title{
INDICADORES
}

\section{SISTEMA DE INDICADORES CULTURALES LOCAL (SICLO)}

\author{
Vicente Coll-Serrano, Salvador Carrasco-Arroyo, Olga Blasco-Blasco y Luis \\ Vila-Lladosa
}
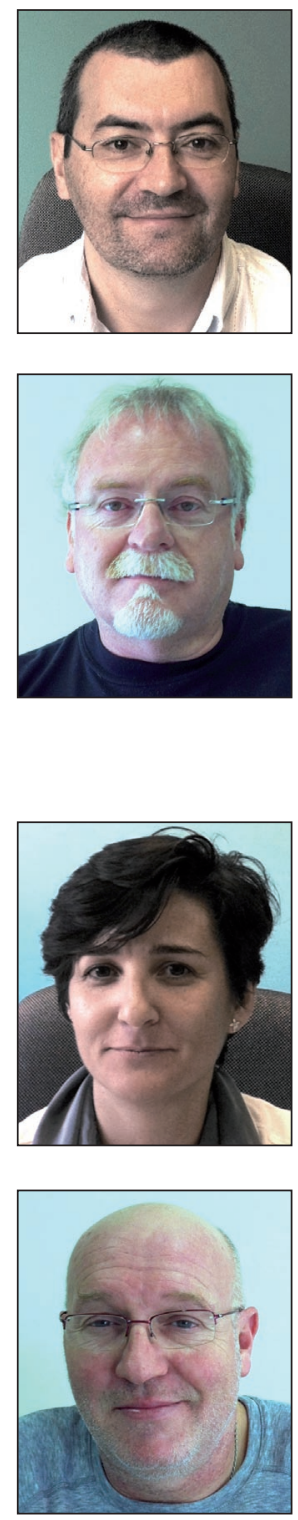

Vicente Coll-Serrano es profesor del Departamento de Economía Aplicada de la Universidad de Valencia y miembro del grupo de investigación MC2 (Métodos Cuantitativos para la Medición de la Cultura). Como investigador ha participado en diversos proyectos y contratos de transferencia vinculados con el diseño e implementación de sistemas de indicadores culturales para distintos organismos y administraciones públicas.

Facultad de Economía Departamento de Economía Aplicada Avda. dels Tarongers, s/n. 46021 Valencia vicente.coll@uv.es

Salvador Carrasco-Arroyo es profesor del departamento de Economía Aplicada de la Universidad de Valencia y director del grupo de investigación MC2 (Métodos Cuantitativos para la Medición de la Cultura). Ha publicado artículos tanto en revistas nacionales como internacionales sobre técnicas de análisis de datos en estudios de fenómenos culturales, educativos. Como investigador principal ha dirigido proyectos sobre diseño de instrumentos de medición, análisis y evaluación de políticas de cultura y desarrollo, y sistemas de información para el seguimiento y evaluación de la acción cultural de entidades públicas.

Facultad de Economía Departamento de Economía Aplicada Avda. dels Tarongers, s/n. 46021 Valencia salvador.carrasco@uv.es

Olga Blasco-Blasco es directora y profesora del Departamento de Economía Aplicada de la Universidad de Valencia. Como miembro del grupo MC2 (Métodos Cuantitativos para la Medición de la Cultura) ha participado como investigadora en el diseño e implementación de sistemas de indicadores para la evaluación de políticas culturales locales. Participa en el proyecto Evaluación de Resultados de Aprendizaje coordinado por el Bologna Experts Team (BET) - España.

Facultad de Economía

Departamento de Economía Aplicada Avda. dels Tarongers, s/n. 46021 Valencia olga.blasco@uv.es

Luis Vila-Lladosa es profesor del Departamento de Economía Aplicada de la Universidad de Valencia, donde imparte cursos de economía de la educación. Es investigador colaborador del Ceges (Estudios en Gestión de la Educación Superior) y miembro del grupo de investigación MC2 (Métodos Cuantitativos para la Medición de la Cultura). Su actividad investigadora y de transferencia se encuentra enfocada a la evaluación econométrica de los efectos socio-económicos de las inversiones educativas tanto desde perspectivas macro como microeconómicas.

Facultad de Economía Departamento de Economía Aplicada Avda. dels Tarongers, s/n. 46021 Valencia luis.vila@uv.es

\section{Resumen}

Se ofrece una visión sintética del proceso que ha dado lugar al Sistema de Indicadores Culturales Local (Siclo), una aplicación orientada a mejorar el conocimiento sobre el estado de la acción cultural en el ámbito de la administración local. Pretende proporcionar información de apoyo que facilite la evaluación de las políticas culturales locales. Se estructura en cinco áreas temáticas y tres módulos. Actualmente sólo se encuentra operativo el módulo básico que está integrado por 46 indicadores.

\section{Palabras clave}

Sistemas de información cultural, Sistema de indicadores, Indicadores culturales, Política cultural, Administración local, Gobierno local, Ayuntamientos.

Artículo recibido el 21-10-11

Aceptación definitiva: 08-12-11 
English title: A local cultural indicators system: Siclo

\section{Abstract}

A synthetic view of the process that led to the Local Cultural Indicators System (Siclo in its Spanish acronym) is presented. Siclo is an application designed to improve knowledge about the status of cultural actions at the local administration level. This tool seeks to provide supporting information to facilitate the evaluation of local cultural policies. It is structured into five thematic areas and three modules. The basic module, comprising a total of 46 indicators, is the only one which is operative at the moment.

\section{Keywords}

Cultural information systems, Indicators systems, Cultural indicators, Cultural policy, Local governments, City councils, Town halls.

Coll-Serrano, Vicente; Carrasco-Arroyo, Salvador; Blasco-Blasco, Olga; Vila-Lladosa, Luis. "Sistema de indicadores culturales local: Siclo". El profesional de la información, 2012, enero-febrero, v. 21, n. 1, pp. 113-117.

http://dx.doi.org/10.3145/epi.2012.ene.15

\section{Introducción}

El concepto de cultura, así como sus implicaciones para el desarrollo económico, social, territorial, urbano y comunitario, ha sido ampliamente debatido en distintos foros. En este documento se delimita la reflexión al ámbito local debido al importante papel que juegan los gobiernos locales en la potenciación del sector cultural como mecanismo de desarrollo. El análisis de la información permitirá apreciar la realidad en un determinado escenario así como investigar las estructuras de las relaciones causales entre cultura y desarrollo. La disponibilidad de información, de datos, es imprescindible para comprender la realidad e intentar mejorarla; la información se revela como el recurso (intangible) más valioso para orientar los cambios.

Sin embargo, el sector de la cultura adolece, en general, de información confiable, homogénea y comparable, lo que dificulta su análisis (Carrasco, 2006). La disponibilidad y el acceso a información suele estar asociado con el grado de desarrollo económico, político y social de un país; aunque cada vez con más frecuencia son las administraciones públicas quienes toman la iniciativa de buscar y acceder a la información cultural y promueven el diseño e implantación de indicadores culturales. Más concretamente, por proximidad a las inquietudes ciudadanas, los gobiernos locales se convierten en actores principales que necesitan estructurar y sistematizar la información cultural a través de un sistema de información confiable y útil que permita satisfacer, por un lado, la necesidad de someter a análisis rigurosos la gestión de las políticas públicas locales y, por otro lado, evaluar la adecuación de la planificación a través del análisis de las relaciones entre los objetivos planificados y los logros alcanzados. Los objetivos últimos del sistema de información son (i) apoyar el proceso de adopción de decisiones para fortalecer la política pública cultural mediante el incremento de la eficacia y la eficiencia en el uso de los recursos y (ii) reforzar los mecanismos de rendición de cuentas de cara a los ciudadanos. Lograr estos objetivos supone mejorar la realidad cultural, que debe ampliar las oportunidades económicas de los individuos y grupos sociales, elevando la renta de las personas y reduciendo la pobreza, a la vez que se mejora la educación, la salud, la conservación del medioambiente, la accesibilidad y participación cultural, así como todas las relaciones simbólicas que se establecen en un escenario complejo y extraordinariamente cambiante como es el urbano (Rausell, 2008).

Establecida la importancia de disponer de un sistema de información cultural a nivel local, en este artículo se describen las principales líneas de trabajo que se han realizado en esta dirección y los resultados alcanzados: el Sistema de indicadores culturales local (Siclo).

\section{Antecedentes}

Para diseñar un sistema de información cultural local es necesario tomar como marco de referencia la Agenda 21 de la Cultura (Ag21) de las Ciudades y Gobiernos Locales Unidos (UCLG, 2004). Éste es el primer documento con espíritu global que apuesta por establecer las bases de un compromiso entre las ciudades y los gobiernos locales para el desarrollo cultural. Según Pascual (2005), es el documento internacional que propone con mayor énfasis un marco normativo para la acción pública en el ámbito de la cultura. Entre sus principales rasgos destacan su valor universal frente a los conflictos que conlleva la globalización cultural, la vocación de transversalidad y la centralidad de las políticas culturales. La Ag21 de la Cultura realiza una serie de recomendaciones a los Estados en materias tan decisivas como los derechos humanos, el medio ambiente y el territorio, la inclusión social y la promoción económica, recalcando el soporte de la cultura como cuarto pilar; pero también recomienda a los gobiernos locales la creación de sistemas de indicadores culturales a partir de los cuales pueda efectuarse el seguimiento y evaluación de las políticas culturales, de sus objetivos y de sus logros.

En base a este marco, en 2005 la Comisión de Cultura de la FEMP en colaboración con el Ministerio de Cultura inició un proyecto para diseñar un sistema de indicadores basado en la necesidad de (i) orientar las políticas culturales locales hacia las tesis planteadas en la Ag21 e (ii) implantar sistemas de información que permitan realizar una planificación estratégica de la política cultural de las administraciónes públicas. 
El resultado de este trabajo se materializó en la Guía para la evaluación de las políticas culturales locales (FEMP, 2009).

Del sistema de indicadores que a nivel teórico se propone en este documento cabe destacar que (i) se centra en la búsqueda de indicadores sencillos, confiables, útiles y conseguibles a nivel local que permitan implantar un sistema de seguimiento y evaluación, y (ii) no contempla todos los principios y recomendaciones que contiene la Ag21 de la Cultura sino sólo las áreas temáticas más relevantes desde el punto de vista de las políticas culturales locales, a saber:

- Cultura y desarrollo;

- Transversalidad de las políticas culturales;

- Cultura y participación ciudadana;

- Accesibilidad de la oferta cultural; y

- Papel de las iniciativas relacionadas con la memoria y la innovación en la construcción de la identidad local.

Bajo el auspicio de la FEMP y con el apoyo del Ministerio de Cultura, a principios de 2010 el grupo de investigación Métodos Cuantitativos para la Medición de la Cultura (MC2) de la Universidad de Valencia se responsabilizó del diseño de una aplicación tutelada que permitiese la implementación efectiva del sistema de indicadores propuesto a nivel teórico en la guía de evaluación (FEMP, 2009). Esta aplicación recibe el nombre de Siclo (Sistema de indicadores culturales local) y actualmente se encuentra en funcionamiento en: http://www.mcmc-uv.es/Siclo http://www.mc2-uv.es/Siclo

En el siguiente apartado se comentan las principales acciones que se han llevado a cabo hasta hacer operativo Siclo.

\section{Sistema de indicadores culturales local}

Siguiendo la filosofía establecida en su antecesora, la guía de evaluación, el Siclo se configura en las siguientes áreas temáticas:

\section{Área A/B. La cultura como factor de desarrollo econó- mico, social y urbano/territorial}

La cultura como factor de desarrollo, en el sentido más amplio del término, es la cuestión central que genéricamente originó y articuló el sistema de indicadores propuesto y hacia la que se orientan las restantes cuestiones. Como se ha dicho, el valor social de la actividad cultural responde a su función como elemento de promoción del desarrollo social y económico de las sociedades y, especialmente en su vertiente local, también del desarrollo territorial. El Siclo aspira a proporcionar información sobre la trayectoria pasada, situación presente y evolución previsible de la cultura. Al mismo tiempo, se considera relevante definir y cuantificar, en su caso, cuáles son los rendimientos, en términos de desarrollo del tejido cultural local, que se derivan de la inversión en cultura, puesto que dichos rendimientos garantizarán la sostenibilidad futura del propio sistema cultural local.

\section{Área C. Transversalidad de la cultura}

La percepción de que la cultura influye en otras políticas locales no relacionadas directamente con la política cultural se ha afianzado en el entorno local debido, fundamentalmente, a la implantación de fórmulas de desarrollo local sostenible, a la importancia estratégica del cambio cultural, y a la consideración de la cultura como instrumento para la proyección exterior de los territorios, instituciones y organizaciones. El Siclo pretende extraer y recopilar información sobre la transversalidad de la cultura desde diversas perspectivas y en distintos niveles. Se aspira a cuantificar la presencia de la cultura en el discurso global de la entidad local, en sus políticas estructurales (social, económica y urbanística) y en ciertas áreas afines (mujer, juventud, participación ciudadana, deportes, turismo) considerando simultáneamente los niveles de transversalidad conceptual, de gestión y de organización.

\section{Área D. Acceso a la cultura}

Siclo aportará información sobre el volumen y naturaleza de las intervenciones encaminadas a favorecer la equidad en el acceso a la oferta cultural pública por parte de todos los ciudadanos $y$, particularmente, sobre el grado de efectividad de dichas intervenciones. Se propone evaluar el alcance de las políticas en términos del grado de desactivación o reducción de las barreras y factores disuasorios que puedan dificultar el acceso de la ciudadanía a la oferta cultural local, sean éstas de índole pecuniaria, de información, de socialización, psicológicas, físicas, cronológicas, de confort o de ausencia de predisposición al hecho cultural.

\section{Área E. Cultura y participación ciudadana}

Este ámbito está estrechamente relacionado con el anterior, en el sentido de que las condiciones de accesibilidad delimitan, a su vez, las posibilidades de participación de los ciudadanos en dos dimensiones principales: como usuarios de servicios culturales locales y como configuradores de la oferta cultural local. Siclo aspira a proporcionar orientación e información sobre el grado de aplicación y, en especial, sobre la efectividad de las acciones de los gobiernos locales dirigidas a incorporar el mayor número posible de personas a la vida cultural local en las dos vertientes anteriormente enunciadas. Para ello se pretende evaluar la situación actual y la evolución futura tanto de los niveles de utilización de los servicios culturales y de asistencia a las actividades culturales como, especialmente, de la implicación y participación activa de los ciudadanos, bien individual o bien colectivamente, en la definición, configuración y gestión de la actividad cultural local.

\section{Área F. Memoria, innovación e identidad}

Este ámbito temático considera, en un contexto crecientemente globalizado, las relaciones entre la memoria colectiva local y la creación contemporánea en el proceso de construcción dinámica de la identidad local. Concretamente, el Siclo obtiene y analiza información sobre la situación del patrimonio cultural, tanto en sus aspectos tangibles como intangibles, entendido como la concreción del proceso de recuperación y proyección de la memoria colectiva. De forma análoga, Siclo también se propone recabar información para el análisis de la vitalidad de la creación cultural endógena y su difusión, puesto que se trata de un elemento clave que interviene en la construcción de la identidad local.

Atendiendo a esta estructura, el diseño y construcción del Siclo arrancó con una propuesta de selección de indicado- 


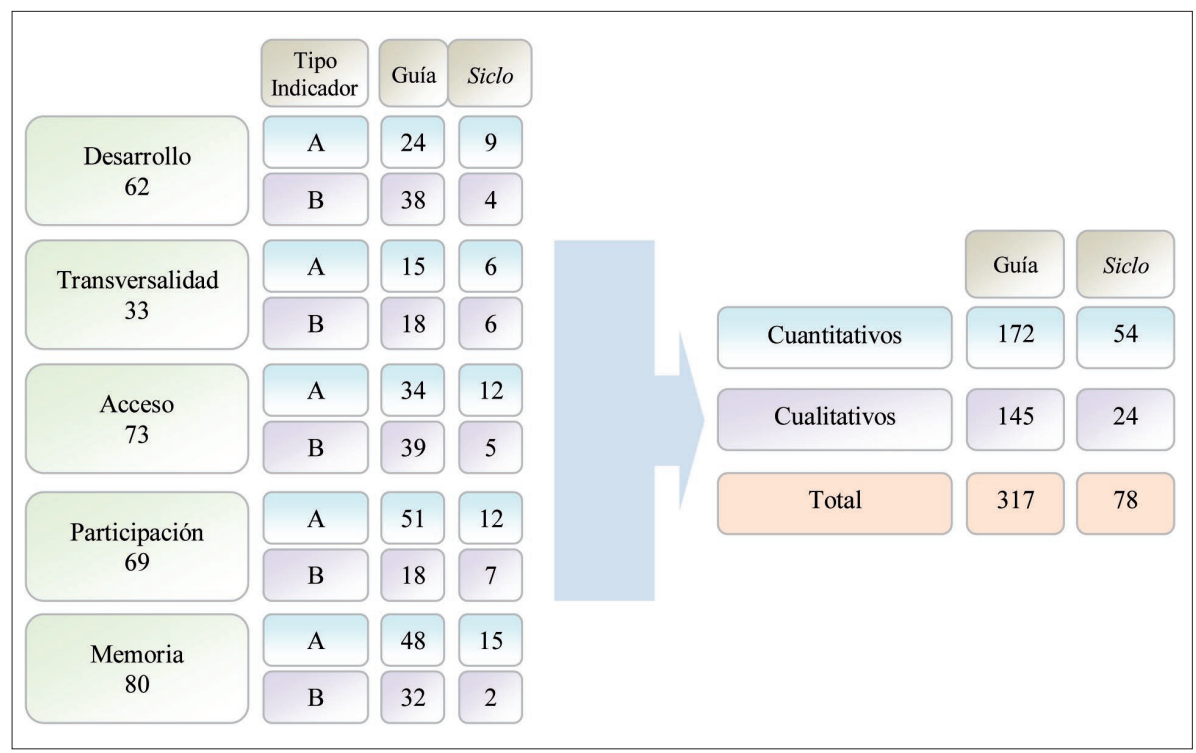

Figura 1. Distribución del número de indicadores. Comparativa guía de evaluación y Siclo. A: Indicadores cuantitativos, B: Indicadores cualitativos

res, puesto que el sistema originalmente estaba formado por un total de 317, lo que resultaba inviable a nivel práctico. Esta selección -basada en el análisis y consenso de un grupo de expertos formado por gestores culturales, académicos y técnicos de cultura tanto de la FEMP como de varios ayuntamientos- condujo a que se retuviese un total de 78 indicadores. La figura 1 muestra la distribución del número de indicadores considerados atendiendo al área de interés y su naturaleza (cuantitativos y cualitativos).

Reducida la dimensión del sistema, se realizó una prueba piloto (pre-test) con el objetivo de contrastar la calidad de los cuestionarios confeccionados para obtener la información requerida, la calidad de los indicadores propuestos y recibir retroalimentación de los responsables de cultura de los gobiernos locales. Para ello se confeccionó un cuestionario telemático que fue cumplimentado por los usuarios potenciales del sistema. En esta fase fueron invitados a participar gobiernos integrantes de la Comisión de Cultura de la FEMP en función de su tipo. Se pretendía que los participantes proporcionasen el mayor volumen posible de información para mejorar los contenidos del instrumento y su operatividad. Los gobiernos que finalmente participaron en la aplicación inicial contribuyeron aportando no sólo los datos de que disponían sobre la cultura y la política cultural en la esfera local sino también numerosos comentarios y sugerencias relativos tanto a los cuestionarios como a los mecanismos de cumplimentación y administración de la información resultante. Esta retroalimentación resultó muy valiosa de cara a la calibración final del sistema de indicadores.

Tras la prueba piloto se realizó un taller de trabajo en la FEMP para analizar los resultados obtenidos. Entre las principales conclusiones a las que se llegó cabe destacar:

- que seguía siendo necesario reducir la dimensión del sistema de indicadores;

- y, sobre todo, que dada la gran heterogeneidad de los gobiernos locales en España era necesario modularlo en función de su tipo.

Así, el Siclo se moduló en tres niveles: Básico, Intermedio y Avanzado. El módulo Básico, núcleo central del sistema, se encuentra integrado por un número limitado de indicadores básicos aplicables a todos los gobiernos locales. De cara al futuro será posible delimitar módulos adicionales (Intermedio y Avanzado) constituidos por grupos de indicadores más específicos que sean de aplicación sólo a determinados municipios en función de determinadas características, por ejemplo de su tamaño.

Actualmente se encuentra operativo el módulo básico del Siclo, que está constituido por un total de 18 indicadores cualitativos y 28 cuantitativos. Debido a restricciones de espacio no es posible abordar con detalle todas las áreas temáticas y todas las cuestiones que en ellas se plantearon a los responsables de las políticas culturales de los gobiernos locales para recabar información. Por esta razón, a modo de ilustración se considera en este artículo sólo el área de Cultura y participación ciudadana (Participación).

El módulo básico de Siclo en el área de Participación está constituido por 10 indicadores: 3 cualitativos (codificados como EQ), que se evalúan en una escala de 1 a 5 , y 7 indicadores cuantitativos (codificados como EN). En esta área se pretende obtener información sobre el uso que los ciudadanos hacen de la oferta cultural promovida por los ayuntamientos así como sobre la actuación de éstos en el fomento de una mayor diversidad de agentes activos (configuradores y gestores de oferta cultural) en la vida cultural local.

Para conocer la demanda efectiva de la ciudadanía con respecto a la oferta cultural promovida por el gobierno local, se pregunta:

- el número de equipamientos culturales, o si existe algún inventario de ellos, distinguiendo los de titularidad o gestión municipal (EQ1a) del resto (EQ1b);

- si existe un sistema integral de recogida de información de la oferta global de actividades y servicios culturales (EQ2);

- si hay canales para que los ciudadanos puedan participar y valorar el diseño de la política cultural (EQ6).

En el aspecto cuantitativo se requiere información, por el lado de la oferta, de:

- butacas/plazas en teatros, auditorios, etc., que son de titularidad, o son gestionados, por el ayuntamiento (EN1);

- metros cuadrados dedicados a actividades de formación cultural y prácticas artísticas (EN2); y

- puestos de lectura/consulta en bibliotecas (EN3).

Y por el lado de la demanda se solicita:

- número de asistentes a las actividades (distinguiendo entre conciertos de música en vivo, representaciones teatrales, danza, etc.), tanto directamente programadas por el gobierno local (EN4a) como de las que reciben su apoyo (EN4b); 
- distribución porcentual de asistentes a los actos escénicos programados o apoyados por la entidad local en 1 año por segmento de público ( $\mathrm{Ni}-$ ños, Jóvenes, Adultos, Personas mayores) y sexo (EN5);

- usuarios del servicio de bibliotecas en 1 año según sexo (EN6); y

- visitantes a museos de titularidad o gestión del ayuntamiento (EN7).

A partir de los datos suministrados voluntariamente por los municipios se procede a la generación de las variables que componen la base de datos general del sistema y la propia de cada usuario. La base de datos general proporciona diversas variables construidas a partir de las respuestas emitidas por los gobiernos locales y constituye el núcleo informativo para el análisis estadístico de los datos y para el proceso de síntesis de la información en un conjunto de indicadores multidimensionales. Los resultados que se derivan del tratamiento de los datos contenidos en el Siclo son facilitados a los usuarios a través de una herramienta diseñada especialmente para ello y que se conoce como Báculo (Barómetro cultural local).

http://www.mc2-uv.es/Baculo

\section{Consideraciones finales}

Las ciudades son los principales espacios de consumo cultural y son los gobiernos locales quienes planifican la política cultural, efectuando el mayor gasto en cultura de la administración pública. Su acción tiene que estar basada en información y análisis y no cabe la toma de decisiones por intuición u olfato político. El Sistema de indicadores culturales local (Siclo) es una aplicación que mejora el conocimiento de la acción cultural en el ámbito de la administración local, lo que facilita la evaluación de las correspondientes políticas. Siclo es el resultado de implementar en la práctica el sistema de indicadores conceptualizado en la Guía para la evaluación de las políticas culturales locales (FEMP, 2009).

De cara al futuro inmediato los principales objetivos que se plantean son incrementar consistentemente el número de ayuntamiento usuarios que alimenten con información el Siclo y mantener el sistema activo en años sucesivos, realizando periódicamente acciones formativas sobre su utilización y potencialidades. En la medida en que se avance en la consecución de estos objetivos será posible generar una secuencia temporal de resultados para cada gobierno local. Tal secuencia constituirá un instrumento fundamental que permitirá a los responsables de las áreas de cultura tanto conocer su posición en relación al estado general de la cultura y de la política cultural, como analizar su trayectoria.

\section{Agradecimientos}

Los autores agradecen la financiación recibida de la Federación Española de Municipios y Provincias así como la colaboración del Ministerio de Cultura de España.

\section{Referencias}

Carrasco-Arroyo, Salvador. "Medir la cultura: una tarea inacabada". Periférica, 2006, n. 7.

http://www.uv.es/carrascs/PDF/medir\%20la\%20cultura.pdf

Ciudades y Gobiernos Locales Unidos (UCLG). Agenda 21 for culture. United Cities and Local Governments, Committee on culture, 2004.

http://agenda21culture.net

Federación Española de Municipios y Provincias (FEMP). Sistema de indicadores para la evaluación de las políticas culturales locales en el marco de la Agenda 21 de la cultura. Madrid, 24 abril 2007.

http://descarga.sarc.es/Curso14/pdf_c/4/4.pdf

Federación Española de Municipios y Provincias (FEMP). Guía para la evaluación de políticas culturales locales. Madrid: FEMP, 2009.

http://www.FEMP.es/files/11-367-fichero/Guia\%20Politicas \%20Culturales.pdf

Pascual, Jordi. Políticas culturales para la diversidad cultural. División de las Políticas Culturales y del Diálogo Intercultural de la Unesco, 2006.

http://www.cities-localgovernments.org/uclg/upload/ newTempDoc/ES_333_report_ex_es.pdf

Rausell, Pau. "Economía de la cultura". Treballs de comunicació, 2008, n. 2, pp. 243-256. 